\title{
AI Technologies and Motives for AI Adoption by Countries and Firms: A Systematic Literature Review
}

\author{
Rene Kabalisa $^{(\bowtie)}$ and Jörn Altmann ${ }^{(凶)}$ \\ Technology Management, Economics and Policy Program, \\ Seoul National University, Seoul, Korea \\ cfmrene@snu.ac.kr, jorn.altmann@acm.org
}

\begin{abstract}
In this era of digital revolution, artificial intelligence stands to be one of the emerging technologies to revolutionize the way we live, work, or communicate. While everyone is fighting to lead in this technology, their readiness differs and adoption challenges arise in many sectors. These competitions also result in various economic impacts on countries, firms, and individuals. This paper uses a systematic literature review to analyze the existing economic impact of AI adoption and the technology used. Overall, this paper presents clear evidence that AI adoption has a large effect on an economy. Findings of this research help researchers and practitioners to identify important economic impacts of adopting AI, identify directions for future research, and set policies that need to be put in place.
\end{abstract}

Keywords: Systematic literature review $\cdot$ Artificial Intelligence $\cdot$ Adoption · Economic impact · AI technology

\section{Introduction}

Countries, firms, and even individuals are economically impacted by technological change. Their decision to adopt a certain technology and its effective use can be crucial to their success. Artificial intelligence (AI) is considered as one of the most time changing technologies in this era of the 4 th industrial revolution. Rao and Verweij projected in 2017 that by 2030 the global GDP will grow by 15.7 trillion US dollars [1].

Artificial intelligence is one of the computer science branches, in which machines can work and respond like humans [3]. In this paper, we consider AI as a combination of technologies that allow machines to act at a high level and enable humans to increase their capabilities in different activities.

Countries are racing in adopting AI technologies. Chen et al. expect that the adoption of AI can directly increase countries' GDP [4]. The adoption of AI in different sectors is expected to make countries become leaders in those sectors, hence increasing that country's economy and hegemony. In 2017, according 
to the Chinese State Council's development plan, China made AI a "new and important" driver of their economy, they expected to become global leaders by 2020, which they have achieved with the AI Chinese industry able to generate around $\$ 59$ billion US Dollars annually [4]. Similarly, other countries have formulated strategic policies related to the adoption and diffusion of AI. Examples are South Korea, Canada, Russia, Philippines, and India.

Not only have countries recognized the importance of AI, but firms also adjusted their business strategies by adopting AI. For example, Google was the pioneer when it formulated the first AI policy followed by Apple. In China, big companies such as Baidu, Tencent, and Alibaba have responded positively by investing in startups that have interest in AI. In that context, small firms chose to adopt $\mathrm{AI}$ in search of new investments.

According to the McKinsey Global Institute report released in 2018, more than $70 \%$ of firms will adopt AI in their businesses by the year 2030. It also predicts that the global economic output production will grow by $16 \%$. In terms of AI market share structure, the latest report by Allied Market research forecasts that the global artificial intelligence market size is expected to reach $\$ 169,411.8$ million by 2025 with a growth rate of $55.6 \%$. The prediction also identifies segments of AI that would lead the market such as machine learning, natural language processing, image processing, and speech recognition.

Furthermore, the large-scale adoption of AI not only generates economic growth to countries and firms but also affects individuals that use that technology every day. So many social implications are being witnessed. Many people are losing their jobs mainly due to the use of AI for automation. In the article about the effect of artificial intelligence on China's labor market, Zhou [8] estimates between 201 and 333 million jobs replaced by either automation or use of robots by 2049 . He also highlights that benefits on individuals will be relatively high compared to the time before AI adoption. For example, considering the acceleration of the aging population, elderly people will be able to physically work with less energy due to the use of AI-controlled robot support. To investigate this cost and benefits in more detail, a review of existing literature would be very helpful.

Therefore, the main objective of this systematic literature review is to investigate the economic impact of AI adoption with respect to identifying the industries impacted, the motivation for adoption, and the AI technology used. The Objective of the research can be expressed in detail with help of the following three research questions $(\mathrm{RQ})$ :

RQ1 What AI technologies impact the most?

RQ2 What motivates countries, firms, or individuals to adopt AI?

RQ3 What are the industries in which the impact of AI adoption is mostly observed?

Subsequent sections of this paper are organized as follows. Section 2 presents the background. Section 3 presents the methodology used, including the details on the systematic literature review protocol. Section 4 presents the analysis and the findings of the review. Section 5 discusses the findings and draws directions for future research. Finally, the conclusion wraps up the key points of the review. 


\section{Background}

In this paper, AI is considered as any type of learning-based technology that is able to increase human performance or replace humans in executing some of their everyday tasks by exhibiting some degree of intelligence or autonomy, if, at the end, they gain a positive economic benefit. AI can be integrated into voice assistants, facial recognition, or embedded in special hardware devices like robots, drones, or Internet of things. Chatbots are AI systems that use natural language processing techniques to conduct a conversation via text or audio. It allows recognizing voices and understanding what is said and being able to respond accordingly. Autonomous machines are defined as a machine that can perform different tasks with no human interventions.

Machine learning is an AI subset that focuses on learning algorithms for machines. It allows machines to learn about new data with no human intervention.

AI is a busy word that is interchangeably used with other terms and words, in this article. The following technical terms will come up very often: automation, mechanization, virtual personal assistance, machine vision, deep learning, NLP, platforms [28], and machine learning.

\section{Methodology}

To conduct the systematic literature review (SLR), this paper adapts the methodology by Webster and Watson [12]. The methodology is explained in the paper "Webster, J., \& Watson, R. T. (2002). Analyzing the past to prepare for the future: Writing a literature review". The Webster and Watson Systematic literature review model identifies relevant literature in a certain field, in order to identify what theories, concepts, and developments are currently discussed in that field. It also helps identifying possible gaps with the aim of developing new theories and new research proposals. Many authors tackling emerging issues on topics such as AI, IoT, Big Data, and Drones use the Webster and Watson model.

Below are the methodological steps that we follow, while conducting this research:

- Step 1: Define topic,

- Step 2: Formulate research questions,

- Step 3: Identify keywords,

- Step 4: Identify search terms,

- Step 5: Identify search in specific databases,

- Step 7: Set selection criteria (i.e., publication types, dates, and languages) and quality assessment criteria,

- Step 8: Collect articles, then use EndNote to organize them,

- Step 9: Reduce the number of articles by applying set of selection criteria, screening,

- Step 10: Fill the data extraction form with the selected papers,

- Step 11: Analyze and document data. 


\subsection{Review Protocol}

The review protocol stores detailed information about the steps applied while searching, selecting, including, and assessing articles to be reviewed in this study. It includes information about:

Research Questions and Keywords: After finishing setting up the topic and the research questions, as laid out in the introduction, keywords are identified that are synonymous to the words used in the research questions.

Search Terms: A manual search was conduced using the terms "artificial intelligence AND adoption", "artificial intelligence AND adoption AND economic impact", "artificial intelligence AND adoption AND benefit"., in order to check the list of terms used.

Databases: The selection of databases comprises the three most frequently used databases by researchers in the technology area. They are Scopus, Web of Science, and Science Direct.

Inclusion Criteria: In order to clearly describe the relevant articles that answers our research questions, we developed a set of inclusion criteria:

- Inclusion criterion 1: As publication date of the research articles, the years between 2000 and 2021 should be selected.

- Inclusion criterion 2: As publication languages, only research articles written in English or French should be selected.

- Inclusion criterion 3: Among the studies obtained, only research articles published in journal articles, conferences proceedings, and early access journals should be selected.

- Inclusion criterion 4: In order to assure quality, articles that comprise an analysis of data and a discussion of results should be selected.

- Inclusion criterion 5: Studies that address the domains of automation, artificial intelligence, and economics should be selected.

Search Queries: Search queries were performed in the three electronic databases using the three search terms. The number of articles obtained after performing the search queries are:

Selection Process: The initial search using the search queries produced a total of 627 articles. After applying the predefined selection criteria (i.e., inclusion criteria), 63 articles were recorded related to automation, AI, and economy. Those 63 articles were read, in order to decide whether those articles are relevance to the research topic and the research questions. Then, the quality of the research articles has been checked, resulting in 30 articles that are used for the systematic literature research.

\section{Analysis of Results}

This section describes the results obtained from our analysis of the selected research articles, organized into a descriptive analysis and findings that answer our research questions (Table 1 ). 
Table 1. Summary of collected articles.

\begin{tabular}{l|l|c}
\hline Search queries & Electronic databases & Records \\
\hline Artificial intelligence AND adoption & Science Direct & 16 \\
& Web of Science & 19 \\
& Scopus & 449 \\
\hline Artificial intelligence AND adoption AND & Science Direct & 7 \\
economic impact & Web of Science & 14 \\
& Scopus & 2 \\
\hline Artificial intelligence AND benefit & Science Direct & 24 \\
& Web of Science & 24 \\
\hline Total & Scopus & 72 \\
\hline
\end{tabular}

\subsection{Descriptive Analysis}

Our findings are based on the results presented in the 30 articles selected. All 30 selected articles were peer-reviewed articles in scientific publications or conference proceedings. Among those 30 articles, 15 were from Web of Science and the other 15 from Scopus. None of our reviewed articles is from Science Direct.

Even though we searched for papers published in year 2000 onwards, those that addressed our research questions started from year 2018. As no other paper could be found that was published before 2018 , it can be stated that the economic impact of AI adoption is still a new topic of research. Moreover, most of the research papers (i.e., 12 of 30 research papers) were published in 2020. As this research was conducted in early June 2021, the number of research papers might increase until the end of the year 2021.

The distribution of selected papers considering countries, in which the AI was adopted, indicates that most research papers on AI adoption focus on emerging and developing countries. Countries like India, China, South-Africa, and Philippines have many research articles on AI adoption.

Regarding the areas of AI adoption, most selected research articles show that AI was adopted in the private sector, followed by a combination of private and public institutions.

Several research methods have been used by research articles selected in our review. Most research articles use surveys though. One of the findings is that most research on AI adoption in countries and firms tend to address practical issues by using surveys. They focus on conceptual framework or policies on how to better adopt and diffuse the use of AI.

\subsection{Economic Impact of AI Adoption}

This section presents the findings with respect to the research questions. The findings address (RQ1) the different types of AI technologies that impact the 
most (Subsect. 4.2.1), (RQ2) the motives of countries and firms for adopting AI (Subsect. 4.2.2), and (RQ3) the industries, in which the economic impact of AI adoption is mostly observed, (Subsect.4.2.3).

\subsubsection{Technologies Adopted}

Figure 1 shows the types of technologies adopted together with AI, according to the reviewed research articles. Although many selected articles only mention AI adoption to reach their goals, some articles are more concrete by mentioning adoptions of combinations of AI with other related technologies. For example, they are highlighted as AI \& automation, AI \& robots, AI with satellites and drones, AI \& cloud computing \& IoT \& blockchain \& big data, automation through robots, as well as Big Data powered with AI.

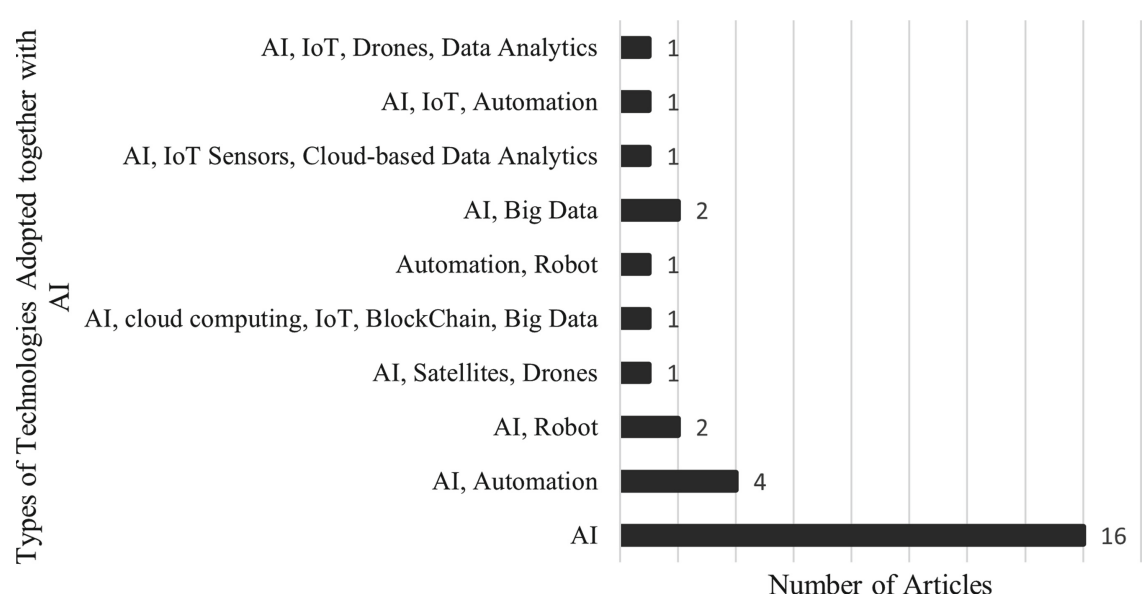

Fig. 1. Distribution of types of technologies adopted from the selected articles.

The AI mentioned in most articles are algorithms and software (e.g., deep learning), which are able to investigate behaviors and support big data analysis. Furthermore, AI robots were also mentioned. It was defined as a combination of machines with software, which is able to act like humans or which is able to be controlled remotely. An example given was electronic vending machines. AI and automation were also mentioned in few articles. It has been identified for cost reduction as well as production growth, but was mentioned mostly in combination with the retail and construction industry.

\subsubsection{Motives for AI Adoption}

The findings from reviewed articles reveal what motivate countries and firms have when adopting AI. The exhaustive list of 13 categories of motives for AI adoption are summarized in Table 2. The most mentioned motivation for AI adoption was to catch up with AI trends and technologies. This motives was given in 10 articles 
$[9,14,18,21,23,25,29,39,41,43]$. Our interpretation is that most articles focused on emerging economies (e.g., China, India, South Africa, and Philippines). These nations are racing to achieve global innovation advantage in AI, in order to catch up with the global leaders (e.g., United States of America, Korea, or Israel) by boosting competitiveness and increasing productivity. The second explanation is that these countries do not want to be left behind in this technology. Support for this is that there are also a few articles with the "catch up" motive that end with designing and formulating "AI policy implementation plans". Examples for that have been articles on India, China, as well as Philippines.

Another major motive that should be highlighted is Pressure to Change. The articles $[15,16]$ address this motive in the context of SMEs in Australia and the wine industry in France. Australian startups and SMEs have a lot of pressure, in order to change the way they serve their clients. Similar issues were identified in the case of the wine industry in France.

The motive "Positioning as a Leader" in AI was also mentioned often in the selected studies. Atwal et al. [4] highlight how the French wine industry, which is currently the global leader, has to adopt AI if it wants to keep its position. As China is competing for global hegemony with the USA in AI [4], it needs to demonstrate leadership. Germany, which is the leader in oil and gas drilling, wants to keep its position as a leader [18] with the help of AI. South Africa that wants to keep its banking sector dominating the African economy [36] by using AI. Similarly, Romania [43] and India [9] want to become leaders in the automobile and health sector with the help of AI.

The motive "Competition" was one of the main motives of AI adoption as well. Nations as well as firms' competitiveness depend on the capacity to innovate $[30,35]$. In this case, many countries and companies chose to adopt AI, increase R\&D funds, or to develop AI policies, in order to be able to compete with other rivals on the global market. In detail, articles were found, which stated the main reason for adopting AI to be: pressure to competition [17]; competitiveness $[9,20,29,39,44]$; competition $[4,23,37,41]$; sustain global competitiveness [32]; increase competitiveness [33]; maintain competitiveness advantages [36]; and get competitiveness advantages [43].

Another motivation for AI adoption, which is related to the private sector, is productivity. India plans to increase productivity in agriculture and health sector [33], the EU attain productivity growth [24], Greece wants to increase productivity efficiency in the transport sector [31], and China envisages to increase productivity and economic growth in almost all sectors [8].

With respect to the motive Country Plan, which is about aligning AI adoption with a country's goals [44], India is a good example. Although India is still far away from AI adoption and diffusion, it aims to attain technological maturity, according to the article of Krishna [32]. For this, the article proposed an AI framework and policy plan for India for almost all sectors of the economy.

Other motives, which are mentioned in the selected articles, are either focused on a single economic aspect or are very general. The motives that were mentioned are Cost [36,38], Customer Acquisition [37], Profit Maximization [37], Saving Money [27], Employment Opportunity [24], and the general motive Economic Benefits [19,20,26,27]. Table 2, summarizes the motives for adopting AI. 
Table 2. Overview of motivation for AI adoption.

\begin{tabular}{|c|c|c|}
\hline Number & $\begin{array}{l}\text { Category of motive for } \\
\text { adopting AI }\end{array}$ & Sub-motives \\
\hline 1 & Catching up & $\begin{array}{l}\text { Catching up with the AI trend (Bello et al. 2019; } \\
\text { Alrashedi \& Abbod 2020; Chatterjee 2020; } \\
\text { Concepcion et al. 2019; Fouda 2020; Hammer \& } \\
\text { Karmakar 2021; Rosales et al. 2020; Somjai et al. } \\
\text { 2020; Srivastava 2018; Turlacu et al. 2018) }\end{array}$ \\
\hline 2 & Pressure to change & $\begin{array}{l}\text { Pressure to change (Alsheibani et al. 2019); external } \\
\text { pressure and organizational readiness (Atwal et al. } \\
\text { 2021) }\end{array}$ \\
\hline 3 & Positioning as a leader & $\begin{array}{l}\text { Positioning as a leader in AI (Atwal et al. 2021); } \\
\text { leader (Bello et al. 2019); be the leader in AI (Chen } \\
\text { et al. 2021); maintain economic advantages } \\
\text { advantages over other countries (Mamela et al. 2020); } \\
\text { market positioning (Srivastava 2018); position itself } \\
\text { in global market (Turlacu et al. 2018) }\end{array}$ \\
\hline 4 & Competition & $\begin{array}{l}\text { Pressure to competition (Bab et al. 2021); } \\
\text { competitiveness (Casalino et al. 2020; Hammer \& } \\
\text { Karmakar 2021; Rosales et al. 2020; Srivastava 2018; } \\
\text { Tyson \& Sauers 2021); competition (Chen et al. } \\
\text { 2021); Concepcion et al. 2019; Nam et al. 2020; } \\
\text { Somjai et al. 2020); sustain global competitiveness } \\
\text { (Krishna 2018); increase competitiveness (Lakshmi } \\
\text { 2020); maintain competitiveness advantages (Mamela } \\
\text { et al. 2020); get competitiveness advantages (Turlacu } \\
\text { et al. 2018) }\end{array}$ \\
\hline 5 & Productivity & $\begin{array}{l}\text { Increase productivity (Lakshmi 2020; Ernst et al. } \\
\text { 2019); attain productivity growth (Ernst et al. 2019); } \\
\text { increase productivity efficiency (Kopsacheilis et al. } \\
\text { 2020); increase productivity and economic growth } \\
\text { (Zhou et al. 2020) }\end{array}$ \\
\hline 6 & Economic benefits & $\begin{array}{l}\text { Perceived economic benefits that come with adoption } \\
\text { of new technologies in business (Bilgeri et al. 2018; } \\
\text { Casalino et al. 2020; Gavrilova \& Gurvitsh-Suits } \\
\text { 2020; Gomes et al. 2020) }\end{array}$ \\
\hline 7 & Country plan & $\begin{array}{l}\text { Align with country's goals and objectives (Tyson \& } \\
\text { Sauers 2021); attain technological maturity (Krishna } \\
\text { 2018) }\end{array}$ \\
\hline 8 & Cost & $\begin{array}{l}\text { Manage workforce by reducing cost (Pan et al. 2021; } \\
\text { Mamela et al. 2020, Lakshmi 2020) }\end{array}$ \\
\hline 9 & Customer acquisition & $\begin{array}{l}\text { Gain customers and market positioning (Nam et al. } \\
2020 \text { ) }\end{array}$ \\
\hline 10 & Profit maximization & $\begin{array}{l}\text { Profit maximization through reducing cost (Lakshmi } \\
2020)\end{array}$ \\
\hline 11 & Saving money & Saving operational expenses (Gomes et al. 2020) \\
\hline 12 & Employment opportunity & $\begin{array}{l}\text { Obtain employment opportunities and impact on jobs } \\
\text { (Ernst et al. 2019) }\end{array}$ \\
\hline
\end{tabular}




\subsubsection{Industries that are Economically Impacted by AI Adoption}

A number of economic impacts of AI adoption were identified in industries. The selected articles covered a wide variety of industry sectors and organizational aspects of firms, in which AI was adopted: governance, SMEs, beverage \& wine, automobile, oil \& gas drilling, automotive, chemistry, firms organizational structure, government, telecom, manufacturing, high tech, agriculture, industrial production, manufacturing, education, transport, distribution, agriculture, accounting, hydrology (dam), transport, healthcare, banking, and tourism. It shows that AI will have an impact in all aspects of our economy.

Several targeted aspects of firms included: increasing performance, cost reduction, increasing sales, competitiveness, production growth, value creation, reducing resources in organizational structure, increasing productivity, and increasing GDP. These aspects are general objectives of a firm [40].

By adopting AI, articles stated that the performance was increased. In some countries after implementing AI policies, their positions on the global markets rose. Examples are China and India. In the case of firms, many firms increased productivity, saved costs and time after adopting AI. Some mentioned improving operating efficiency and solving many optimization problems.

\section{Discussion and Conclusion}

In this paper, a systematic review of existing literature from 2000 to 2021 on the economic impact of AI adoption by countries and firms has been presented. We carried out a systematic literature review identifying relevant research articles that answered our research questions from Scopus, Web of Science, and Science Direct databases, eventually resulting in 627 papers. After applying selection criteria, 63 papers were considered for cross-reading and quality checking. After this process, 30 articles remained for our final review.

All research articles in our sample were published in the last four years. Most research papers in our sample focus on emerging and developing countries and also show that the majority of the AI adoption happened in firms. This paper also identified different technologies that were adopted in combination with AI, those are 1) AI \& automation, 2) AI \& robots, 3) AI with satellites and drones, 4) AI \& cloud computing, 5) automation \& robots, and 6) big data powered with AI.

Referring to research questions, this paper identifies the motives of AI adoption. The most repeated motives were catch up, pressure to change, positioning as a leader, competition, increase productivity, reduce cost, other perceived economic benefits, attain technological maturity, align with the country's goals, market positioning, and saving operational expenses.

We were expecting cases of papers about AI adoption by certain individuals. Many jobs will be carried out by machines, software's, or robots, in this sense AI adoption can affect workers. We couldn't find any specific article explaining which jobs would be lost or which new job opportunities were created by the AI adoption and how it impacted certain individuals. 
Most cases were either AI adoption by countries or firms. A number of economic impacts were also identified, including increasing performance, cost reduction, increased sales, competitiveness, production growth, value creation, reduced resources in organizational structure, increased productivity, and increased GDP. This confirms that AI is a technology that can be adopted across different sectors of the economy. Deep and more research on economic adoption of AI in different industries (financial, government, ...) are proposed for future research. Our review couldn't find articles related to the economic impact of AI adoption on individuals. The economic impact of AI adoption by individuals mostly on employment or any cases where AI can make individuals' life simpler or relaxed is proposed for future studies. Which jobs are to be lost as well as which industry to be affected by AI adoption and which industry is to produce new jobs will be good research questions. Also, research on AI best practices is needed, most of our research is from countries where the AI index is low, further research about the economic impact on countries with higher AI index is recommended.

Furthermore, research on economic factors that led to the adoption of AI as well as economic factors that challenges the Adoption of AI is proposed for future studies. Today, it is hard to measure the positive effect of AI adoption or the negative effect of AI adoption. We would like to propose a kind of performance measure for AI adoption for future studies.

Finally, we will provide tools and technologies used for the adoption and diffusion of AI which can benefit individuals looking for new skills and new jobs opportunities.

Acknowledgements. This research was supported by the BK21 FOUR (Fostering Outstanding Universities for Research) funded by the Ministry of Education (MOE, Korea) and National Research Foundation of Korea (NRF). This work was also supported by the National Research Foundation of Korea (NRF) grant (No. NRF2019R1F1A1058487) funded by the Ministry of Science and ICT (MSIT) of Korea.

\section{References}

1. Jan, S.T., Ishakian, V., Muthusamy, V.: AI trust in business processes: the need for process-aware explanations. In: Proceedings of the AAAI Conference on Artificial Intelligence, vol. 34, pp. 13403-13404 (2020)

2. Brown, N., Sandholm, T.: Superhuman AI for heads-up no-limit poker: Libratus beats top professionals. Science 359, 418-424 (2018). https://doi.org/10.1126/ science.aao1733

3. Miller, S.: Computer Scientist Coined 'Artificial Intelligence'. WS (2011)

4. Chen, H., Li, L., Chen, Y.: Explore success factors that impact artificial intelligence adoption on telecom industry in China. J. Manag. Anal. 8, 36-68 (2021)

5. Dutton, T.: An overview of national AI strategies. Politics+ AI (2018)

6. Hall, O.P., Virtue, J.: Artificial intelligence techniques enhance business forecasts: computer-based analysis increases accuracy. Graziadio Bus. Rev. 5, 4-8 (2002)

7. Simon, J.P.: Artificial intelligence: scope, players, markets and geography. Digital Policy, Regulation and Governance (2019) 
8. Zhou, G., Chu, G., Li, L., Meng, L.: The effect of artificial intelligence on China's labor market. China Econ. J. 13, 24-41 (2020)

9. Srivastava, S.K.: Artificial Intelligence: way forward for India. JISTEM-J. Inf. Syst. Technol. Manage. 15, 4-20 (2018)

10. Bughin, J., Seong, J., Manyika, J., Chui, M., Joshi, R.: Notes from the AI frontier: modeling the impact of AI on the world economy. McKinsey Global Institute (2018)

11. Frey, C.B., Osborne, M.A.: The future of employment: how susceptible are jobs to computerisation? Technol. Forecast. Soc. Chang. 114, 254-280 (2017)

12. Webster, J., Watson, R.T.: Analyzing the past to prepare for the future: writing a literature review. MIS Q. 26, xiii-xxiii (2002)

13. Wiles, J.: Action plan for $\mathrm{HR}$ as artificial intelligence spreads. Gartner report (2018)

14. Alrashedi, A., Abbod, M.: The effect of using artificial intelligence on performance of appraisal system: a case study for University of Jeddah Staff in Saudi Arabia. In: Arai, K., Kapoor, S., Bhatia, R. (eds.) IntelliSys 2020. AISC, vol. 1250, pp. 145-154. Springer, Cham (2021). https://doi.org/10.1007/978-3-030-55180-3_11

15. Alsheibani, S.A., Cheung, D., Messom, D.: Factors inhibiting the adoption of artificial intelligence at organizational-level: a preliminary investigation (2019)

16. Atwal, G., Bryson, D., Williams, A.: An exploratory study of the adoption of artificial intelligence in Burgundy's wine industry. Strateg. Chang. 30, 299-306 (2021)

17. Bag, S., Pretorius, J.H.C., Gupta, S., Dwivedi, Y.K.: Role of institutional pressures and resources in the adoption of big data analytics powered artificial intelligence, sustainable manufacturing practices and circular economy capabilities. Technol. Forecast. Soc. Chang. 163, 120420 (2021)

18. Bello, O., Teodoriu, C., Oluwafemi, O., Olayiwola, O.: Successful Geothermal Operation Management: Technology Adoption of Oil and Gas Drilling Rig Systems

19. Bilgeri, D., Fleisch, E., Wortmann, F.: How the IoT affects multibusiness industrial companies: IoT organizational archetypes (2018)

20. Casalino, N., Saso, T., Borin, B., Massella, E., Lancioni, F.: Digital competences for civil servants and digital ecosystems for more effective working processes in public organizations. In: Agrifoglio, R., Lamboglia, R., Mancini, D., Ricciardi, F. (eds.) Digital Business Transformation. LNISO, vol. 38, pp. 315-326. Springer, Cham (2020). https://doi.org/10.1007/978-3-030-47355-6_21

21. Chatterjee, S.: AI strategy of India: policy framework, adoption challenges and actions for government. Transforming Government: People, Process and Policy (2020)

22. Chung, H.: Adoption and development of the fourth industrial revolution technology: features and determinants. Sustainability 13, 871 (2021)

23. Concepcion, R.S., Bedruz, R.A.R., Culaba, A.B., Dadios, E.P., Pascua, A.R.A.R.: The technology adoption and governance of artificial intelligence in the Philippines. In: 2019 IEEE 11th International Conference on Humanoid, Nanotechnology, Information Technology, Communication and Control, Environment, and Management (HNICEM), pp. 1-10. IEEE (2019)

24. Ernst, E., Merola, R., Samaan, D.: Economics of artificial intelligence: implications for the future of work. IZA J. Labor Policy 9, 1-35 (2019)

25. Fouda, T.: Impact of the fourth industrial revolution on the development of scientific research in the field of agricultural engineering in Egypt and Arab World. Scientific Papers Series-Management, Economic Engineering in Agriculture and Rural Development, vol. 20, pp. 253-258 (2020) 
26. Avrilova, V., Gurvitsh-Suits, N.A.: Contemporary innovation challenges-future of adoption artificial intelligence: case of Estonia. Eur. Integr. Stud. 14, 217-225 (2020)

27. Gomes, M.G., et al.: Economic, environmental and social gains of the implementation of artificial intelligence at dam operations toward Industry 4.0 principles. Sustainability 12, 3604 (2020)

28. Haile, N., Altmann, J.: Evaluating investments in portability and interoperability between software service platforms. Futur. Gener. Comput. Syst. 78, 224-241 (2018). https://doi.org/10.1016/j.future.2017.04.040

29. Hammer, A., Karmakar, S.: Automation, AI and the future of work in India. Empl. Relat. Int. J. 43, 1327-1341 (2021)

30. Kim, K., Altmann, J.: Platform provider roles in innovation in software service ecosystems. IEEE Trans. Eng. Manage., 1-10 (2020). https://doi.org/10.1109/tem. 2019.2949023

31. Kopsacheilis, A., Nikolaidou, A., Georgiadis, G., Politis, I., Papaioannou, P.: Investigating the prospect of adopting artificial intelligence techniques from transport operators in Greece. In: Nathanail, E.G., Adamos, G., Karakikes, I. (eds.) CSUM 2020. AISC, vol. 1278, pp. 1097-1106. Springer, Cham (2021). https://doi.org/10. 1007/978-3-030-61075-3_105

32. Lakshmi, V.: Exploring autonomous vehicle technology - a case study for the Indian automotive industry (2018)

33. Lakshmi, V., Bahli, B.: Understanding the robotization landscape transformation: a centering resonance analysis. J. Innov. Knowl. 5, 59-67 (2020)

34. Lee, C.S., Tajudeen, F.P.: Impact of artificial intelligence on accounting: evidence from Malaysian organizations. Asian J. Bus. Account. 13, 214-222 (2020)

35. Lee, W., Yoon, J., Altmann, J., Lee, J.-D.: Model for identifying firm's product innovation dynamics: applied to the case of the Korean mobile phone industry. Technol. Anal. Strateg. Manag. 33, 335-348 (2020). https://doi.org/10.1080/ 09537325.2020.1813271

36. Mamela, T.L., Sukdeo, N., Mukwakungu, S.C.: The integration of AI on workforce performance for a South African Banking Institution. In: 2020 International Conference on Artificial Intelligence, Big Data, Computing and Data Communication Systems (icABCD), pp. 1-8. IEEE (2020)

37. Nam, K., Dutt, C.S., Chathoth, P., Daghfous, A., Khan, M.S.: The adoption of artificial intelligence and robotics in the hotel industry: prospects and challenges. Electron. Mark. 31, 1-22 (2020)

38. Pan, Y., Froese, F., Liu, N., Hu, Y., Ye, M.: The adoption of artificial intelligence in employee recruitment: the influence of contextual factors. Int. J. Hum. Resour. Manag. 25, 1-23 (2021)

39. Rosales, M.A., Jo-ann, V.M., Palconit, M.G.B., Culaba, A.B., Dadios, E.P.: Artificial intelligence: the technology adoption and impact in the Philippines. In: 2020 IEEE 12th International Conference on Humanoid, Nanotechnology, Information Technology, Communication and Control, Environment, and Management (HNICEM), pp. 1-6. IEEE (2020)

40. Shim, D., Kim, J.G., Altmann, J.: Strategic management of R\&D and marketing integration for multi-dimensional success of new product developments: an empirical investigation in the Korean ICT industry. Asian J. Technol. Innov. 24, 293-316 (2016). https://doi.org/10.1080/19761597.2016.1253023

41. Somjai, S., Jermsittiparsert, K., Chankoson, T.: Determining the initial and subsequent impact of artificial intelligence adoption on economy: a macroeconomic survey from ASEAN. J. Intell. Fuzzy Syst. 39, 1-16 (2020) 
42. Szalavetz, A.: Artificial intelligence-based development strategy in dependent market economies-any room amidst big power rivalry? Cent. Eur. Bus. Rev. 8, 40-54 (2019)

43. Chivu, R.G., Orzan, M., Turlacu, L.M., Radu, A.V.: Alternative evaluation methods in university education. eLearn. Softw. Educ. 4, 7-12 (2018)

44. Tyson, M.M., Sauers, N.J.: School leaders' adoption and implementation of artificial intelligence. J. Educ. Admin. 59, 229-234 (2021)

45. Markram, H.: The human brain project. Sci. Am. 306, 50-55 (2012)

Open Access This chapter is licensed under the terms of the Creative Commons Attribution 4.0 International License (http://creativecommons.org/licenses/by/4.0/), which permits use, sharing, adaptation, distribution and reproduction in any medium or format, as long as you give appropriate credit to the original author(s) and the source, provide a link to the Creative Commons license and indicate if changes were made.

The images or other third party material in this chapter are included in the chapter's Creative Commons license, unless indicated otherwise in a credit line to the material. If material is not included in the chapter's Creative Commons license and your intended use is not permitted by statutory regulation or exceeds the permitted use, you will need to obtain permission directly from the copyright holder.

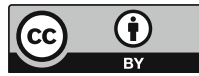

